\title{
Ratio and interconnections of functional fitness structure key components of elite combat athletes at the stage of maximum realization of individual capabilities
}

\author{
Oleksander Pryimakov $^{1 \mathrm{ACDE}}$, Sergii Iermakov ${ }^{2 \mathrm{CDE}}$, Jerzy Eider $^{3 \mathrm{ADE}}$, Stanislav Prysiazhniuk $^{4 \mathrm{ADE}}$, Anatoly Skrypko ${ }^{5 \mathrm{DE}}$, \\ Natalija Mazurok ${ }^{5 \mathrm{DE}}$ \\ ${ }^{1.3}$ Institute of Physical Culture Sciences, Szczecin University, Poland \\ ${ }^{2}$ Gdansk University of Physical Education and Sport, Gdańsk, Poland \\ ${ }^{4}$ State University of Telecommunications, Kyiv, Ukraine \\ ${ }^{5}$ State University of Applied Sciences in Kalysz, Poland \\ ${ }^{6}$ National Pedagogical Dragomanov University, Kyiv, Ukraine
}

Authors' Contribution: A - Study design; B - Data collection; C - Statistical analysis; D - Manuscript Preparation; E - Funds Collection

\section{Abstract}

Purpose:

The objective of the work consisted in studying the ratio and interconnections of functional fitness structure key components of elite combat athletes in the process of adaptation to physical loads at the stage of individual capacity maximum realization.

Material: $\quad 55$ highly skilled wrestlers aged 19-27 years old, members of the national teams of Ukraine in different styles of wrestling - freestyle, Greco-Roman, judo were examined. The assessment of wrestlers' functional fitness structure was made according to a complex of indices of physiological systems activity at rest and during performance of a block of testing physical loads on a bicycle ergometer.

Results: $\quad$ The components of functional fitness structure of male combat athletes of three wrestling styles (GrecoRoman, freestyle and judo) were studied according to the manifestations of the respiratory and circulatory functions during testing loads performance, and achieved indices of work capacity. It has been revealed that the key integrative components of the general structure of wrestlers' fitness include: the level of special work capacity, general level of functional fitness, mobility of physiological processes, economy, anaerobic power. Greco-Roman wrestlers are superior to freestyle wrestlers and judokas in anaerobic power, mobility and economy. The greatest differences are manifested in the values of anaerobic power, mobility and general level of functional fitness, whereas the least - in indices of economy. The key criteria for the functional fitness of wrestlers in the manifestation of a high level of physical work capacity include: economy of functioning at rest and during muscular activity of anaerobic-aerobic nature, high reactivity of the cardiovascular system to physical load, power of the anaerobic energy supply system, the rate of recovery processes, the state of cardiac activity regulatory mechanisms, the level of metabolic processes and excitability of the heart muscle. The level of development and the ratio of the components of functional fitness structure of elite combat athletes may be used as the reference models for development of differentiated scales for estimation of the key constituents of physical state of wrestlers specialized in freestyle, Greco-Roman wrestling and judo. Complex application of different methods of testing athletes provides more efficient approach to the problem of monitoring and managing functional fitness and special work capacity of wrestlers at the stage of maximum realization of individual capacities.

Conclusions: An increase in the proportion of key integrative functional indices in special work capacity of athletes specialized in various wrestling styles - Greco-Roman, freestyle and judo represent the most significant criterion for improving their functional fitness structure.

Keywords: $\quad$ wrestlers of different styles, structure, functional fitness, criteria, work capacity.

\section{Introduction}

Efficient studying functional fitness structure (FFS) of combat athletes, the variety of geno- and phenotypic factors that determine its formation in the process of long-term adaptation to physical loads, is possible on the basis of complex research methods and principles of a systematic approach [1-4].

The main provisions and principles of systematic approach envisage the study of the ratios of physiological parameter development levels and the interconnections

c Oleksander Pryimakov, Sergii lermakov, Jerzy Eider, Stanislav Prysiazhniuk, Anatoly Skrypko, Natalija Mazurok, 2020 doi:10.15561/20755279.2020.0505 of mechanisms determining special fitness of athletes at different stages of the training process [5-8].

Despite the fact that many researchers used complex research methods to study the integral activity of the body of combat athletes, the structure of athletes 'fitness was analyzed without account for the ratio of development levels and interconnections of its key components to ensure the athletes' special work capacity [9-11].

Most authors analyzed the general structure of combat athlete fitness, mainly according to its individual components, factors, or few indices without taking into account their ratio and interconnections in the process 
of ensuring specific activity of wrestlers [12-15]. Few complex studies failed to observe the main principle of the systematic approach - the relationships and ratios of the constituents of structural and functional components in the general fitness structure of athletes were incompletely taken into account in connection with ensuring a high level of special work capacity or competitive activity [16$18]$.

Insufficient coverage of functional fitness structure of combat athletes from systemic positions prevented the authors from substantiating a differentiated approach to its assessment in wrestlers of different weight categories, age, skill level, gender, style of wrestling [19, 20].

This made it difficult to assess the structure of combat athlete functional fitness as one of the key components of their general fitness structure, along with physical, technical, psychological and moral-volitional fitness. In addition, it also reduced the effectiveness of methodological approaches aimed at the formation and improvement of athlete fitness structure in the process of long-term adaptation to physical loads.

The study of physiological systems interconnected activity in the structure of functional and general fitness of athletes is necessary for substantiated and efficient managing adaptation reorganizations, increasing the level of special work capacity, reliability of performing motor actions and the growth of sports results in wrestling [8, $21,22]$.

The importance of this problem for the theory and practice of wrestling, system physiology, physiology of physical education and sport, and its insufficient coverage in the literature have determined the choice of direction and subject of study.

The objective of this work is to study the ratio and interconnections of functional fitness structure key components of elite combat athletes in the process of adaptation to physical loads at the stage of individual capacity maximum realization.

\section{Material and methods}

Participants. 55 highly skilled wrestlers aged 19-27 years old, members of the national teams of Ukraine in different styles of wrestling - freestyle, Greco-Roman, judo were examined. The 31 of Ukrainian National Team athletes, participated in this study, were at the student's age (19-24 years old) during championships and 24 of them were currently students.

Study protocol was approved by Ethic Committee University. The research was fulfilled in compliance with WMA Declaration of Helsinki - Ethical Principles for Medical Research Involving Human Subjects [23].

Procedure. The studies were carried out at different stages of the annual preparation cycle and performed as a natural open experiment.

To assess the functional fitness (FF) structure of combat athletes, instrumental research methods as well as methods of mathematical statistics, mathematical modeling and forecasting were used.

The structure of athlete functional fitness was examined in a state of a relative rest and during responses to testing physical loads.

Vegetative and somatic indices were recorded in a state of a relative rest by electrocardiography and variational pulsography methods [24], tremometry, computer-based testing $[6,22]$, wrist and torso dynamometry, methods for recording the maximum, relative and explosive strength of the lower extremity muscles [6], etc.

Parameters of the electrocardiogram, time of simple and complex motor responses, accuracy of visual information processing, frequency of movements in the tapping test, speed-strength manifestations of the lower extremity muscles, and other indices were recorded at rest under basal conditions. AsCARD MrGrey v.07.205 electrocardiograph of ASPEL S.A (Poland) company was used to record temporal and amplitude characteristics of the electrocardiogram.

The assessment of wrestler functional fitness structure during muscular activity was made according to the indices of the activity of the muscular, respiratory and cardiovascular systems and the achieved work capacity indices while performing the block of testing loads on the bicycle ergometer. Various test conditions allowed to evaluate the general level of athlete functional fitness by the degree of development of its key structural properties, such as anaerobic and aerobic power, mobility and economy [21, 25]. This structuration of the body functional capacities enabled to outline the most generalized properties of FFS, which are subjected to targeted improvement through the use of specially selected training effects [25].

The "Oxycon Pro" high-speed automatic gas analyzer of "Jeager" company (Germany) was used to examine the body functional manifestations. In the process of performing loads, the following initial indices were determined: respiratory minute volume $\left(1 \cdot \mathrm{min}^{-1}\right)$ concentration of oxygen and carbon dioxide in exhaled air [\%), heart rate [beats $\cdot \min ^{-1}$ ) and the corresponding indices of the power of performed load [W). According to the results of performing the battery of testing loads, a complex of 9 indices was calculated, based on which such key factors of functional fitness structure as anaerobic power, mobility, economy was differentiated in accordance with specially designed algorithm $[5,25$, 26]. General level of functional fitness and the degree of development of the studied structural properties were determined on the basis of a system of formalized ballpoint assessment of indices [5].

The examinations were carried out on a "Monark" bicycle ergometer [Sweden). The following types of testing loads were used: 1) State of rest - 3 minutes; 2) Load of standard [moderate) power -6 min [2 W per kg of weight); 3) Anaerobic power load lasting 30 seconds; 4) Anaerobic power load lasting 60 seconds.

Recovery pauses between testing loads were filled with unloaded pedaling.

Statistical analysis. STATISTICA 13,5 statistical package was used for statistical processing of experimental material [27]. 


\section{Results}

As a result of conducted studies, it was found that in a state of relative rest, under basal conditions (in the morning, lying down after waking up), the characteristic signs of a high level of the body functional state of elite combat athletes include: according to electrocardiography and variational pulsography - bradycardia (heart rate (HR)) from 38 to 52 beats $\cdot \mathrm{min}^{-1}$ ), increase in QRS complex duration without signs of blockade, increased voltage of the T wave in leads V3 - V5, an upward 1-2 $\mathrm{mm}$ shift of the ST segment in leads V1, V2, increase in duration and variability of the R-R interval, respiratorytype arrhythmia, decrease in Baevsky myocardial stress index (SI) [24].

These characteristic signs of athlete's heart are most apparent among lightweight wrestlers. They reflect a more pronounced economy of their heart and the body functioning.

In wrestlers of higher weight categories, heart rate and SI are higher than those of lightweight athletes. They also have the greatest increase in heart rate and SI when performing an orthostatic test.

Statistical processing of vegetative and somatic indices recorded under basal conditions, as well as while performing a number of tests in a state of relative rest, allowed to determine the key generalized factors and indices of wrestlers' FFS: 1) factor of the optimal ratio and interconnections of physiological mechanisms determining the general level of wrestlers' physical state ( $46.7 \%$ of the total variance of the studied indices); 2 ) factor of economy and differentiation of regulatory mechanisms during manifestation of muscle speedstrength capacities (36.0\% of the total variance) 3 ) factor of interconnections between the components of vegetative support and central-nervous coordination in controlling local movements (14.7\% of the total variance).

The following vegetative indices were determined as the key ones: heart rate, myocardial SI, arterial pressure (AP), hemoglobin $(\mathrm{Hb})$ content. The key somatic indices include: the tremor amplitude, frequency of movements in the tapping test, strength of the right and left hands, strength and ratio of excitation and inhibition processes in the central nervous system, static endurance, maximum, relative and explosive strength of the lower extremity muscles, the time of simple and complex motor responses, accuracy of visual information processing.

The use of analysis-of-variance methods allowed to show that, in a state of relative rest, the variability of FFS vegetative and somatic indices is more determined by the body weight of athletes than their skill level.

Higher weight categories are associated with increased activity of cardiovascular system, higher tension of functioning of cardiac activity regulatory mechanisms (according to SI), elevated diastolic $(\mathrm{P}<0,01)$ and decreased pulse $(\mathrm{P}<0,01) \mathrm{BP}$, improved absolute strength capacities of athletes $(\mathrm{P}<0,01)$, reduced relative strength of trunk extensor muscles $(\mathrm{P}<0,01)$, decreased strength of inhibitory process in $\mathrm{CNS}(\mathrm{P}<0,01)$.

Of the somatic parameters, the most variable at rest is the tremor amplitude reflecting the state of the neuromuscular system and regulatory mechanisms that coordinate the extremity micromotions while controlling the wrestler vertical posture stability.

Evaluation of the structure of wrestler functional fitness general level by manifestations of respiratory and circulatory functions in response to ongoing testing loads and achieved work capacity indices $[5 ; 21]$ allowed to determine the level of development and the ratio of the key components of FFS of combat athletes, such as anaerobic and aerobic power, mobility and economy.

Table 1 shows statistical expression of the factors of functional fitness structure of combat athletes for the manifestation of their athletic work capacity (table 1).

The following ratios of the key factors in the general structure of combat athlete functional fitness were revealed without account for their specialization: anaerobic power - 53,0 $\pm 2,1 \%$, aerobic power - 53,5 $\pm 1,8 \%$, mobility - 80,4 $\pm 2,8 \%$, economy $-45,3 \pm 2,5 \%$. The results are indicative of high mobility level and relatively low economy of physiological processes in combat athletes. The level of anaerobic and aerobic power is characterized by average values.

The most integral index - the general level of functional fitness (FF), determined by mobility, economy, anaerobic and aerobic power, is at an average level of $55 \pm 2.0 \%$ in the combined group of combat athletes (Table 1).

Below are presented mathematical models of the general level of wrestlers' functional fitness dependence on the level of development and the interconnections of the integral components of FF structure (Table 2).

Table 1. Factors of functional fitness structure of combat athletes according to manifestations of respiratory and circulatory functions during testing loads

\begin{tabular}{llllll}
\hline \multirow{2}{*}{$\begin{array}{l}\text { Statistical } \\
\text { parameters }\end{array}$} & $\begin{array}{l}\text { Factors of functional fitness structure } \\
\text { Anaerobic power, }\end{array}$ & $\begin{array}{l}\text { Aerobic power, } \\
\%\end{array}$ & $\begin{array}{l}\text { Mobility, } \\
\%\end{array}$ & $\begin{array}{l}\text { Economy, } \\
\%\end{array}$ & General level of FF, \% \\
\hline $\bar{x}$ & $\mathbf{5 3 , 0}$ & $\mathbf{5 3 , 5}$ & $\mathbf{8 0 , 4}$ & $\mathbf{4 5 , 3}$ & $\mathbf{5 5}$ \\
$\pm \mathrm{m}$ & 2,1 & 1,8 & 2,8 & 2,5 & 2,0 \\
$\mathrm{~S}$ & 15 & 13 & 20 & 17 & 15 \\
$\mathrm{~V}, \%$ & 28,5 & 24,6 & 24,9 & 39,3 & 28,3 \\
$\mathrm{n}$ & 53 & 52 & 51 & 50 & 55 \\
\hline
\end{tabular}


Regression models reflect the share contribution of individual integral indices of the functional fitness structure of Greco-Roman, freestyle, and judo wrestlers to the general structure of their functional state.

Revealed models are the basis for development of a system for assessing and predicting the wrestlers' FFS, differentiated according to the level of development of its key integral components: anaerobic power, economy, aerobic power, mobility. Both in the combined group of highly skilled combat athletes and in each separate group of subjects: Greco-Roman, freestyle, and judo wrestlers.

Figure 1 shows the components of functional fitness structure of male combat athletes representing three styles of wrestling (Greco-Roman, freestyle and judo) according to the manifestations of respiratory and circulatory functions under testing loads and achieved indices of work capacity.

Comparative analysis of FFS of highly skilled representatives of different wrestling styles indicates that Greco-Roman wrestlers are superior to freestyle wrestlers and judokas in the following components of functional fitness: anaerobic power, mobility and economy.
The greatest differences are manifested in the values of anaerobic power and mobility.

Statistically significant differences reflect more expressed ability of the body of Greco-Roman wrestlers to faster restore functions after physical load, (mobility), and to achieve the highest levels of work capacity under short-term physical load (anaerobic power).

The least differences between three groups of wrestlers were manifested in indices of economy, reflecting the ability of athletes to perform physical loads with a minimum level of function strain and minimal energy expenditures.

Figure 2 illustrates the general level of functional fitness of highly skilled freestyle and Greco-Roman wrestlers and judokas.

Average values of functional fitness general level, as an integral index determined by mobility, economy, anaerobic and aerobic power, are higher in GrecoRoman wrestlers $(60.1 \pm 3.2 \%)$. Freestyle wrestlers and judokas are inferior to Greco-Roman wrestlers in terms of functional fitness level $(40.2 \pm 2.8 \%$ and $39.0 \pm 2.9 \%$, respectively), which probably reflects the specifics of

Table 2. Mathematical models of the general level of wrestlers' functional fitness dependence on the levels of development and ratio of its integral components

\begin{tabular}{lll} 
Groups of subjects & Regression models & Correlation coefficients (R) \\
\hline $\begin{array}{l}\text { Combined group of } \\
\text { combat athletes }\end{array}$ & $Y=\left(\left(0,295 x_{1}+0,248 x_{2}+0,280 x_{3}+0,046 x_{4}\right)-0,749\right) \pm 6,11$ & $R=0,938, p<0,0000 .$. \\
Greco-Roman wrestling & $Y=\left(16,26+0,281 x_{1}+0,337 x_{2}+0,044 x_{3}+0,055 x_{4}\right) \pm 6,2$ & $R=0,903, p<0,00000$. \\
Freestyle wrestling & $Y=\left(21,6+0,019 x_{2}+1,11 x_{3}-0,253 x_{1}-0,533 x_{4}\right) \pm 0,92$ & $R=0,993, p<0,00000 .$. \\
Judo & $Y=\left(21,1+0,271 x_{2}+0,366 x_{3}+0,082 x_{4}-0,208 x_{1}\right) \pm 4,7$ & $R=0,943, p<0,00000 .$. \\
\hline
\end{tabular}

Notes: $\mathrm{Y}$ - general level of FF, $\%$; $\mathrm{x}_{1}$ - aerobic power; $\mathrm{x}_{2}$ - anaerobic power, $\% ; \mathrm{x}_{3}$ - mobility; $\mathrm{x}_{4}$ - economy.

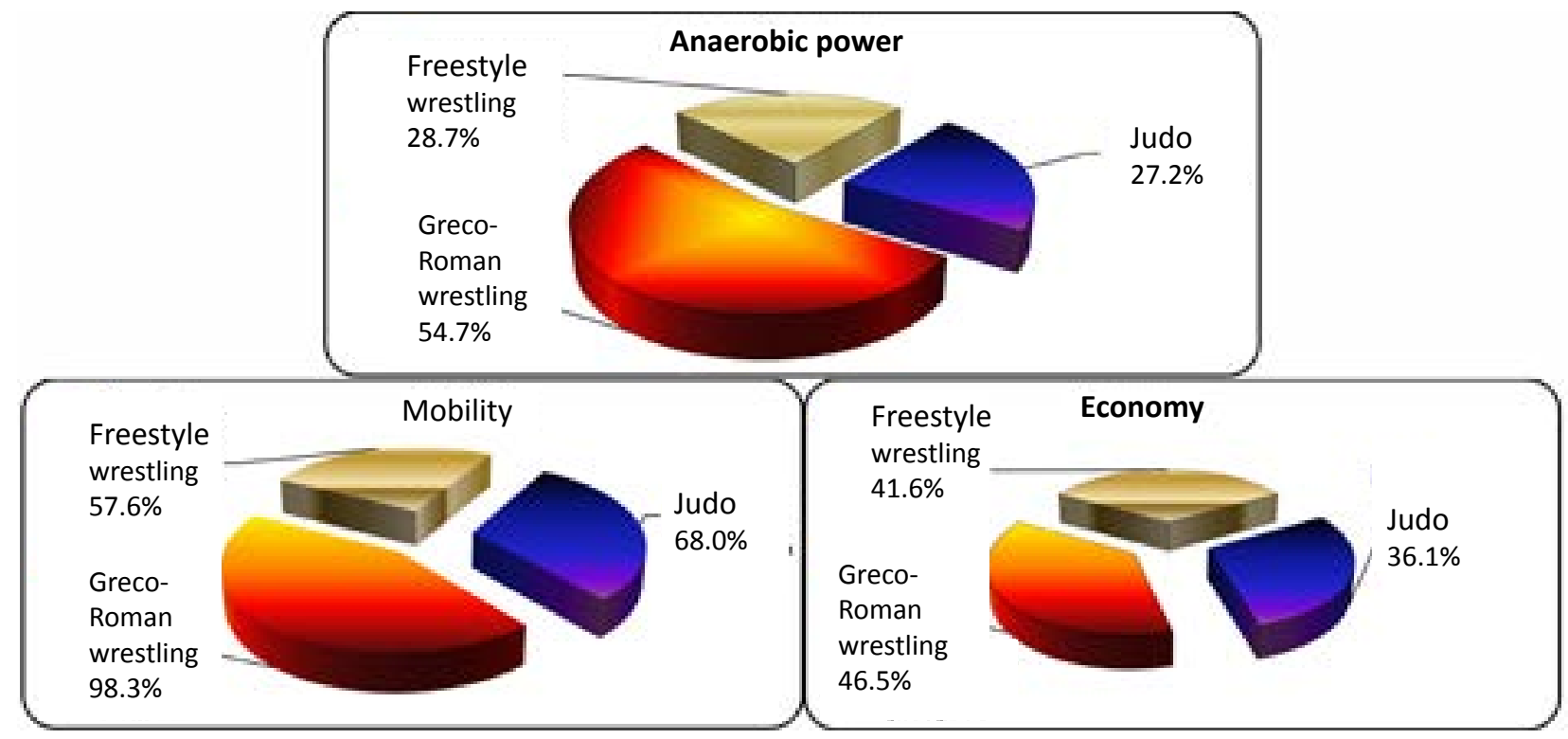

Fig. 1. Ratio of the components of functional fitness structure in athletes specialized in judo, freestyle and GrecoRoman wrestling 


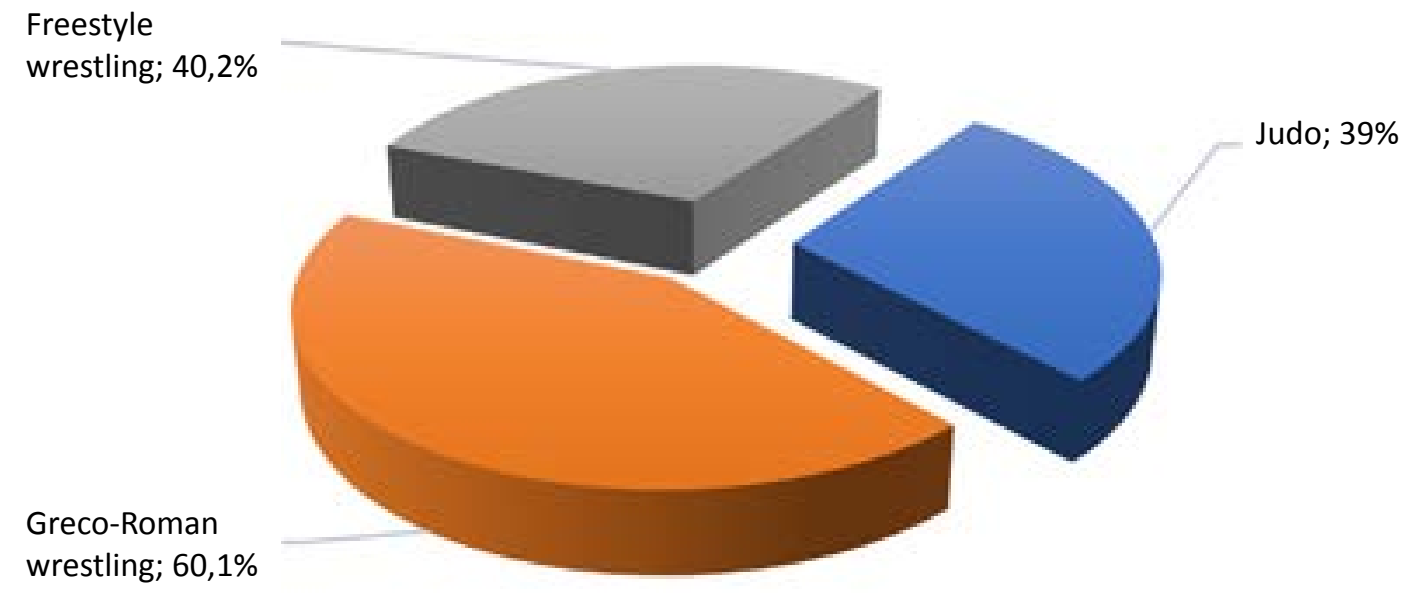

Fig. 2. General level of functional fitness (\%) in highly skilled wrestlers of different specialization (freestyle and GrecoRoman wrestling, judo)

adaptive morphofunctional alterations associated with distinctive peculiarities of technical and competitive preparation of combat athletes of various specializations. Verification of this assumption may become the subject of further in-depth studies.

The results indicate that Greco-Roman wrestlers are most functionally prepared as compared to freestyle wrestlers and judokas, according to the outlined criteria.

Therefore, elite athletes of the analyzed groups differ both in the general level of functional fitness and in the level of development of the studied structural properties. The greatest differences are manifested in the parameters of anaerobic power, mobility and integral index - the general level of functional fitness.

\section{Discussion}

The dominance of the analytical approach in studying the structure of combat athlete fitness allowed researchers to uncover different aspects of athletes' physical state in the process of long-term adaptation to physical loads [10, 12, 28-30]. This approach, however, prevented them from examining the mechanisms of athlete body integration systems in the process of providing the high level of their fitness at different stages of training process. Application of the system approach methods in various sports events enabled some researchers to analyze the specifics of several body system integration in the process of ensuring a high level of physical work capacity $[6,25]$.

This approach permitted to evaluate not only the level of development, but also the ratio and interconnections of the components of athletes' fitness general structure in the process of modeling different modes of muscular activity energy supply [7, 18, 25].

However, most of the similar studies dealt with the analysis of functional fitness structure of athletes specialized mainly in cyclic sports events $[25,26]$. At the same time, FFS of combat athletes is reflected in special literature on wrestling without account for the variety of interdependent factors affecting its formation: weight category, gender, age, skill level and other factors $[6,8]$.

Conducted studies resulted in obtaining experimental material that characterizes the structure of combat athlete functional fitness according to complex of vegetative and somatic indices in various physiological states: before and under different physical loads, which show the role of various energy systems in ensuring physical performance of combat athletes specialized in different wrestling styles - judo, freestyle and Greco-Roman.

The use of complex research methods, including the cyclic test block, allowed us to carry out complex registration of physiological indices at rest, as well as under different modes of muscular activity, with accurate recording parameters of load, respiration, cardiac activity and work capacity $[22,31]$.

Complex registration of the parameters of combat athlete FFS provided the framework for developing appropriate models of systemic support for the muscular activity of highly skilled wrestlers. Previously developed models $[6,8,31,32]$, supplemented by the results of the given study, allow to differentiate the assessment of functional fitness structure of wrestlers according to different criteria and in various physiological states of their body (rest, muscular activity).

At the same time, while evaluating and interpreting the findings, one should take into account the fact that, according to the structure of movements, the cyclic load is not specific to acyclic activity of combat athletes.

In the studies of various authors, as well as in our earlier works, combat athletes were also tested using exercises that model their specific activity. This allowed to make indirect quantitative [in terms of time and number of movements performed in the test) and qualitative [in terms of quality of specialized movements technique) assessment of the level of their special work capacity and fitness [6, 31]. However, the degree of precision of the estimates of FFS, the ratio of its components, and the level of physical work capacity during such test execution is lower than during performing a block of cyclic loads.

The use of a block of physical loads of varying intensity and duration with registration of cardiac activity and respiration parameters provides high-precision determination of the level of development and the ratio 
of the components of athletes' functional fitness structure, the character of energy supply, as well as the level of their non-specific work capacity $[4,6,31]$.

High mobility of the body functional manifestations of highly skilled wrestlers reflects its ability to restore functions as soon as possible after physical load [25, 26].

Differentiation of the estimates of the various FFS components of highly skilled combat athletes of different weight categories during performance of the testing load block allows high-precision assessment of their functional reserves, manifested differently in responses to physical load at various stages of annual training cycle [31]. It also permits to differentiate monitoring of physical state and fitness control of wrestlers of light and heavy weight categories according to key indices of cardiac activity of gas analysis and special work capacity [31-34].

\section{Conclusions.}

Generalization of the findings allows to believe that the key criteria for the functional fitness of highly skilled wrestlers providing a high level of physical work capacity include: economy of physiological systems functioning at rest and during muscular activity of anaerobic-aerobic nature, high reactivity of the cardiovascular system to physical load, power of the anaerobic energy supply system, rate of recovery processes, state of cardiac activity regulatory mechanisms, level of metabolic processes and excitability of the heart muscle.

The level of development and the ratio of the components of functional fitness structure of highly skilled combat athletes may be used as the reference models for development of differentiated scales for estimation of the key constituents of physical state of wrestlers specialized in freestyle, Greco-Roman wrestling and judo. Complex application of different methods of testing athletes provides more efficient approach to the problem of monitoring and managing functional fitness and special work capacity of wrestlers at the stage of maximum realization of individual capacities.

\section{Conflict of interests.}

The authors declare that there is no conflict of interest.

\section{References}

1. Anokhin PK. Key issues of functional system theory. Moscow: Science; 1980. (In Russian).

2. Damiani APL, Caldas LC, Melo AB, Entringer CD, Estevam $\mathrm{WM}$, Nogueira BV, et al. Resistance traning protocols promote strength increase without morphological changes. Revista Brasileira De Medicina Do Esporte. 2020;26(3):253-257. https://doi.org/10.1590/1517-869220202603209955

3. Isaev AP, Erlikh VV, Bakhareva AS, Saraykin DA, Pavlova VI, Maleev DO. Effects of short- and longterm adaptation to the middle-altitude hypoxia on the condition of athletes practicing cyclic and acyclic sports. Minerva Ortopedica E Traumatologica, 2018;69(3):31-42. https://doi.org/10.23736/s0394-3410.18.03873-0

4. Pryimakov AA, Osipenko AA, Kolenkov AV, Dan'ko TG. Control for functional fitness of elite wrestlers at precompetitive stage of preparation. Pedagogics, Psychology, Medical-Biological Problems of Physical Training and Sports, 2004;3: 96-102. (In Russian).

5. Mischenko V. Physiological monitoring of athletic training: current approaches and directions of improvement. Nauka $v$ Olimpiyskom Sporte, 1997; 1(6): 92-103. (In Russian).

6. Kolenkov OV, Pryimakov OO, Prystynskyi VM, Osiptsov AV. Modeling structure of special physical fitness of highly skilled wrestlers at the stage of maximum realization of individual capacities. Donetsk: Knowledge; 2012. (In Russian).

7. Pryimakov AA, Erzy Eider, Kolenkov AV. Managing educational and training process on the basis of fitness model characteristics of highly skilled combat athletes. Science and Education. 2016; 8: 142-150. (In Russian). https://doi.org/10.24195/2414-4665-2016-8-28

8. Pryimakov OO. Comparative characteristics structure physically prepared fighters high qualification light, medium and heavy weight category. Pedagogics, Psychology, Medical-Biological Problems of Physical Training and Sports, 2014; 9: 47-53. (In Russian). https://doi.org/10.5281/zenodo.10128

9. Anthierens A, Olivier N, Thevenon A, Mucci P. Trunk Muscle Aerobic Metabolism Responses in Endurance
Athletes, Combat Athletes and Untrained Men. International Journal of Sports Medicine, 2019;40(7):434-439. https://doi.org/10.1055/a-0856-7207

10.Theophilos P, Ioannis B, Nikolaos M, Athanasios K, Konstantinos M, Bahman M. Physiological Profile Evaluation Through Lactate and Heart Rate in National Level Greco-Roman Wrestlers. International Journal of Wrestling Science, 2011;1:68-72. https://doi.org/10.1080/21615667.2011.10878935

11. Sagiroglu I, Kurt C, Pekunlu E, Ozsu I. Residual effects of static stretching and self-myofascial-release exercises on flexibility and lower body explosive strength in well-trained combat athletes. Isokinetics and Exercise Science, 17;25(2):135-141. https://doi.org/10.3233/ies-160656

12.Kalina RM, Jagiello W. Non-apparatus, Quasi-apparatus and Simulations Tests in Diagnosis Positive Health and Survival Abilities. In: Ahram T, editor. Advances in Human Factors in Sports, Injury Prevention and Outdoor Recreation, Ahfe 2017. Advances in Intelligent Systems and Computing. 6032018. p. 121-128.

13.Oleksy M, Kalina RM, Mosler D, Jagiello W. Quasiapparatus shime waza test (QASWT) - validation procedure. Archives of Budo. 2018;14:133-147.

14.Burdukiewicz A, Pietraszewska J, Stachon A, Andrzejewska J. Anthropometric profile of combat athletes via multivariate analysis. Journal of Sports Medicine and Physical Fitness. 2018;58(11):1657-1665. https://doi.org/10.23736/s0022-4707.17.07999-3

15.Maksutova GI, Popova TV, Romanov YN, Surina-Marysheva EF,KourovaOG.Psychophysiologicalstatusofmenandwomen in combat sports. Human Sport Medicine, 2020;20(2):63-70. https://doi.org/10.14529/hsm200208

16.Kelleher LK, Beach TAC, Frost DM, Johnson AM, Dickey JP. Factor structure, stability, and congruence in the functional movement screen. Measurement in Physical Education and Exercise Science. 2018;22(2):109-115. https://doi.org/10.1080/1091367x.2017.1381608

17.Boyko VF, Danko GV. Physical preparation of wrestlers. Kiev: Olympic Literature; 2004. (In Russian). 
18.Suarez GR, Velasquez CAA. Proposal for the evaluation of the sports form in judo. Viref-Revista De Educacion Fisica. 2020;9(1):1-28.

19.Calmet M. Developing ecological re-search in judo. Perceptual and Motor Skills, 2007; 105(2): 646- 648. https://doi.org/10.2466/pms.105.2.646-648

20.Pryimakov AA. Activity and relationships of muscular and cardiovascular systems in different states during muscular activity in athletes. Physical Education of Students, 2012; 6: 93-99. (In Russian). https://doi.org/10.6084/m9.figshare.96576

21.Mishchenko VS. Functional potentials of sportsmen. Kiev: Health; 1990. (In Russian).

22.Pryimakov A, Driukov V. Improvement of the system of complex control for functional fitness of highly skilled athletes. Nauka v Olimpijskom Sporte, 2004; 1: 61-67. (In Russian).

23.World Medical Association Declaration of Helsinki: Ethical Principles for Medical Research Involving Human Subjects. JAMA, 2013;310:2191. https://doi.org/10.1001/jama.2013.281053

24.Bayevskiy RM, Motylyanskaya RYe. Cardiac rhythm in athletes. Moscow: Physical Culture and Sport; 1986. (In Russian).

25.Mishchenko VS, Lysenko EN, Vinogradov VE. Reactive features of cardiorespiratory system as the reflection of adaptation to strenuous physical training in sport. Kiev: Scientific World; 2007. (In Russian).

26.Lysenko OM. Physiological reactivity and peculiarities of mobilizing functional capacities of highly skilled athletes. Aktualni Problemy Fizychnoi Kultury i Sportu, 2003; 1: 8186. (In Russian).

27.Borovikov VP, Ivchenko GI. Prognostication in the system of
Statistica in the environment of Windows. Moscow: Finances and statistics; 2006. (In Russian).

28.Kraemer William. The Physiological Basis of Wrestling: Implications for Conditioning Programs. The Strength and Conditioning Journal, 2004; 26(2): 10-15. https://doi.org/10.1519/00126548-200404000-00001

29.Rahmani-Nia F, Mirzaei B, Nuri R. Physiological profile of elite Iranian junior Greco-Roman wrestlers. International Journal of Fitness, 2007; 3: 49-54.

30.Erkan Demirkan. The monitoring of weight fluctuation and hydration status in cadet wrestlers (ages 14-17) during a training camp period leading up to competition. International Journal of Wrestling Science, 2011; 1(2): 12-18. https://doi.org/10.1080/21615667.2011.10878925

31.Pryimakov O, Iermakov S, Kolenkov O, Samokish I, Juchno J. Monitoring of functional fitness of combat athletes during the precompetitive preparation stage. Journal of Physical Education and Sport, 2016; 16(2): 551 -561. https://doi.org/10.7752/jpes.2016.0208

32.Pryimakov O, Iermakov S, Eider J, Prysiazhniuk S, Mazurok N. Physiological criteria of functional fitness and determinants of physical work capacity of highly skilled wrestlers. Physical Education of Students, 2020; 24(4): 205-212. https://doi.org/10.15561/20755279.2020.0403

33. Romanenko V, Podrigalo L, Cynarski WJ, Rovnaya O, Korobeynikova L, Goloha V, et al. A comparative analysis of the short-term memory of martial arts' athletes of differentlevelofsportsmanship. IdoMovementforCultureJournal of Martial Arts Anthropology. 2020;20(3):18-24. https://doi.org/10.14589/ido.20.3.3

34. Yushkov OP. Improvement of training methods and complex control in combat sports events. Proceedings of the VI International Research Congress "Olympic Sport and Sport for All". Warsaw. 2002; 46 (1,2): 135-136.

\section{Information about the authors:}

Oleksander Pryimakov; (Corresponding author); http://orcid.org/0000-0003-0351-486X; alprim7491@gmail.com; Faculty of Physical Culture and Health Promotion, Szczecin University; Szczecin, Poland.

Sergii lermakov; Doctor of Pedagogical Sciences, Professor; http://orcid.org/0000-0002-5039-4517; sportart@gmail.com; Department of Sport, Gdansk University of Physical Education and Sport; Gdansk, Poland.

Jerzy Eider; http://orcid.org/0000-0002-8401-6442; sekretariat.wkfipz@univ.szczecin.pl; Faculty of Physical Culture and Health Promotion, Szczecin University; Szczecin, Poland.

Stanislav Prysiazhniuk; Professor; http://orcid.org/0000-0002-3017-0268; stas046@ukr.net;University ofTelecommunications, Kiev, Ukraine.

Anatoly Skrypko; https://orcid.org/0000-0002-6472-6535; anskrypko@wp.pl; State University of Applied Sciences in Kalisz; Kalisz, Poland.

Natalija Mazurok; https://orcid.org/0000-0001-7346-1156; natprim75@gmail.com; National Pedagogical Dragomanov University, Kyiv, Ukraine.

\section{Cite this article as:}

Pryimakov O, lermakov S, Eider J, Prysiazhniuk S, Skrypko A, Mazurok N. Ratio and interconnections of functional fitness structure key components of elite combat athletes at the stage of maximum realization of individual capabilities Physical Education of Students, 2020;24(5):286-292.

https://doi.org/10.15561/20755279.2020.0505

This is an Open Access article distributed under the terms of the Creative Commons Attribution License, which permits unrestricted use, distribution, and reproduction in any medium, provided the original work is properly cited http://creativecommons.org/licenses/by/4.0/deed.en 